\author{
Military Technical College \\ Kobry El-Kobbah, \\ Cairo, Egypt.
}

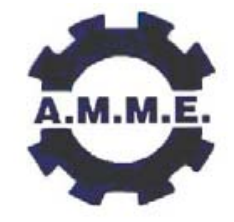

\title{
THE EFFECT OF AIR LATCH ON THE AIRFLOW IN 3.5-INCH HARD DISK DRIVE
}

\author{
$13^{\text {th }}$ International Conference \\ on Applied Mechanics and \\ Mechanical Engineering.
}

\author{
BOONYARITCHAIKIT P., $, *, * * *$, TAECHAJEDCADARUNGSRI S. ${ }^{* * * * *}$ \\ and HORMDEE D."
}

\begin{abstract}
Nowadays, the performance of computers has improved very fast. The design development of data storage devices such as Hard Disk Drive (HDD) has also improved in order to gain more capacity with less access time. Therefore, the higher spindle motor speed is needed. This requirement will then directly affect to the airflow inside the HDD, which is one of the major factors causing the vibration on the recording head whilst flying on the data track. The objective of this paper is to study on the airflow in 3.5-inch with 7200-rpm motor speed, single head HDD, one of the most common HDD models in the current market. Three-dimensional RNG K-epsilon Model will be used in this study by comparing the airflow of two different models: the former is the model with air latch and the latter is the model without air latch. The Head Gimbal Assembly (HGA) position at Outside Disk (OD) position and Inside Disk (ID) position are also studied. The simulation results with RNG K-epsilon Model showed that by repositioning the HGA, the airflow inside the HDD is also changed. The airflow inside the HDD across the arm and the HGA is significant faster when the HGA is positioned at OD. Therefore, the arm and the HGA at OD position will face the vibration problem higher than at the ID position. The air latch is found to reduce air velocity about $35 \%$. The conclusions of this paper would be useful for further study on flow induce vibration on the HGA.
\end{abstract}

\section{KEY WORDS}

Hard Disk Drive (HDD), RNG Kepsilon, Head Gimbal Assembly (HGA), Air latch

\footnotetext{
* Researcher, Industry/University Cooperative Research Center (I/UCRC) in HDD Component, Khon Kaen University, Khon Kaen 40002, Thailand.

** Graduate student, Dpt. of Mechanical Engineering, Khon Kaen University, Khon Kaen 40002, Thailand.

*** Staff, Seagate Technology (Thailand).

**** Assistant Professor, Dpt. of Mechanical Engineering, Khon Kaen University, Khon Kaen 40002, Thailand. (Corresponding Author: sirtae@kku.ac.th)

${ }^{* * * * *}$ Assistant Professor, Dpt. of Computer Engineering, Khon Kaen University, Khon Kaen 40002, Thailand.
} 


\section{INTRODUCTION}

Hard Disk Drives (HDD) in personal computers normally are 3.5-inch platform. When operated, the disk in HDD spin at its constant speed. The air latch is used to hold the actuator arm at its parking position when the drive does not operate and to release the arm when the drive starts to operate. Once the disk begins to spin, the effect of air circulation around the disk triggers the air latch to release the arm into operating area. While the arm and the head fly on the disk, they are always disturbed by the airflow. The pressure and velocity of the airflow has significant influence on the vibration of the recording head. The numerical studies of the airflow behavior inside HDD using Large Eddy Simulation (LES) has been reported [1]. In 2003, H. Song et al. [2] studied the flow field and particle trajectories in HDD, using three different turbulence models; standard K-epsilon, RNG K-epsilon and Reynolds Stress Method. M.A. Suriadi et al. [3] studied airflow in 1-inch HDD with acceptable results by using standard K-epsilon turbulence model. Y. Hirono et al. [5] studied on flow-induced vibration Reduction in HDD using a Laser Dropper Vibrometer (LDV) to measure amplitude of the vibration. And flow-induced vibration using LDV and static pressure sensor to measure in actual HDD has also been researched [6]. This study focuses on the numerical simulation of airflow inside 3.5-inch HDD. Four cases have been simulated regarding the position of the actuator arm whether it is located at Inside Disk (ID) and Outside Disk (OD) positions. These four simulations are:

- Actuator arm at ID position with air latch (IDwithAL)

- Actuator arm at ID position without air latch (IDw/oAL)

- Actuator arm at OD position with air latch (ODwithAL)

- Actuator arm at OD position without air latch (ODw/oAL)

\section{SIMULATION MODEL OF HARD DISK DRIVE}

The three-dimensional model is constructed based on the actual design of 3.5 -inch HDD available in the market, as shown in Fig. 1.

The major components of the model in this study are disk, head, base and air latch. When HDD operates, the disk will be rotated counter clockwise at a specific rotating speed and the head will be moved in and out for data read/write. This simulation model is based on the single head HDD with 7200 rpm disk rotation speed. In order to study airflow, all fluid inside the HDD is considered as the air.

In this study, the commercial FLUENT software is employed to simulate the airflow inside HDD. Due to the limitation and the conversion of the results on the software, also compared with the elements used on 3.5 inch HDD in [4], the 1.3 million elements are used. Within an acceptable calculation time on a personal computer, the RNG KEpsilon turbulence model is used instead of other turbulent models such as Reynolds Stress Model (RSM), Large Eddy Simulation (LES) and Direct Numerical Simulation (DNS) which are time consuming and require lots of computer resources [3-5].

In boundary conditions, the disk and the spindle motor (Fig. 2) are set as moving walls with the rotating speed of $7200 \mathrm{rpm}$. 


\section{RESULTS AND DISCUSSION}

Simulation results have been categorized into 3 sections; study on static pressure, study on air velocity and study on airflow pattern in HDD.

\section{Study on Static Pressure}

To study the static pressure for each simulation case, a HDD is horizontally cut into three planes as in Fig. 3: the plane below (A), middle $(B)$ and above $(C)$ of actuator arm.

Figures 4, 5 and 6 illustrate static pressure of all three cut planes with four cases (IDwithAL, IDw/oAL, ODwithAL and ODw/oAL) for each plane. Considering the models without air latch for all three different cut planes (Figs. 4, 5 and 6), the simulation results show that the static pressure is about 10 pa at OD and -7 pa at ID. This is because the airflow is blocked by the actuator arm. On the other hand, the static pressure at the bottom of HDD around the voice coil is about 20 pa.

For the model with air latch, the pressure profile has changed with 50 pa. for the pressure acting on the air latch. The three cutting planes all show the same pressure profile, which is the higher pressure when adding the air latch into the HDD. The results, for both with and without air latch, also show that the static pressure around disk area for the ID case is generally higher than for the OD case.

Figure 7 along with Table 1 emphasize on the maximum of static pressure at the actuator head.

\section{Study on Air Velocity}

Air velocity of the disk surface is verified by comparing the results from solid surface velocity of the rotating disk. The left of Fig. 8 illustrates that velocity will increase as the radius of the disk increases. While the right graph of Fig. 8 shows that the velocity at the center of the disk is the lowest and the highest velocity is at the outer rim with 35.8 $\mathrm{m} / \mathrm{s}$. This value is about the same as what of the calculation from the rotating velocity equation (1) where $v$ is the velocity, $N$ is the rotating speed and $r$ is the radius of the disk.

$$
v=\frac{N}{60} \times 2 \pi r \quad(m / s)
$$

Figures 9, 10 and 11 illustrate air velocity of all three cut planes with four cases (IDwithAL, IDw/OAL, ODwithAL and ODw/oAL) for each plane. The results show that the overall air velocity decreases when the cut plane height increases. The simulations point out that the perturbation of airflow velocity on the head when the actuator arm at OD position is higher than when the actuator arm at ID position. This is because the air velocity at the inner side of the disk is lower than the outer side. Hence, the pressure fluctuation causes the head vibration at OD position easier than at ID position. Considering the model with air latch, when the arm is at OD position, the air velocity at the back of the air latch is dropped. Thus the velocity of airflow against the arm is decreased from $11.2 \mathrm{~m} / \mathrm{s}$ to $7.0 \mathrm{~m} / \mathrm{s}$. However, the velocity around the recording head 
still remains at the same order of magnitude. While the arm is at ID position, the air velocity around the recording head is slightly increased due to the airflow across the air latch. Moreover, there is another air circulation moving from the extended arm of the air latch back into inner disk area. Therefore, the lowest air velocity area is shifted from below motor shaft to the left of the motor shaft due to the existing of the air latch.

Figure 12 along with Table 2 emphasize on the maximum of air velocity at actuator head.

\section{Study on the Airflow Pattern}

The simulations of airflow pattern between OD and ID actuator head positions are illustrated in Fig.13.

For the model without air latch, the air flows away outward from the center of the disk until it flows against the wall around the disk. Then the air flows to the open wall (at the lower left corner by the disk). The air will flow outward from the disk through this open wall. The small vortex is found in this area when the actuator arm is located in OD position but it disappears when the actuator is located at ID position. After that the air will flow toward into disk area again at the open wall at the right corner.

From the airflow profile of the model with air latch, the air stream flows against the air latch and moves along the air latch arm toward the inner side of the disk. The airflow that moves pass the air latch arm flows toward the HDD arm and recording head. Some part of the airflow move through the opening path around the backside of the HDD, behind the voice coil, and flows toward the right corner of the disk. This airflow generates the vortex at the lower right side of the HDD. The air circulates around HDD right corner and then flows back into the rotating disk area with irregular airflow profile. The further study of the airflow without air latch is to investigate the flow on the vertical cut plane along the actuator arm (Fig. 14: section 1). There is an air vortex behind the voice coil on both OD and ID of arm positions. However, there is no air vortex behind the voice coil according to the results of the model with air latch in Fig. 15.

The airflow from the cut plane at the middle and the tip of the actuator arm, as shown in Fig. 14: section 2-3, has shown that when the actuator is located at OD position, the airflow without the air latch moves outward with the direction pointing perpendicular to the cut plane (Fig. 14: section 3) and moves around the voice coil toward the right corner of HDD with the direction pointing parallel along the arm to the head tip.

With the air latch, the air flows from the inner side of the disk across the arm to the outer area and creates a small vortex (perpendicular to the cut plane) beside the arm. When the arm is at ID position without air latch, the air flows across the arm to the outer area without creating any vortex beside the arm, as shown in Figs.16 and 17.

\section{CONCLUSIONS}

This study concerns the effect of the air latch on the airflow in 3.5-inch HDD with the constant rotating speed at $7200 \mathrm{rpm}$. When the actuator arm is at the OD position, there is inward-outward flow creating the small vortex near the outer side of the disk. Flow velocity and the static pressure at this point is higher than when the arm is at the 
ID position. The result of airflow velocity is reduced by $35 \%$ when the air latch is applied to the model. The maximum pressure occurs when the actuator arm is at the OD position. And the pressure acting on the arm is higher when the air latch is used. The fluctuation of pressure along the arm induces head and arm vibrations. The study of airflow pattern shows that small vortex locates close to the arm when the arm moves to OD position. The strength of small vorticity affects the fluctuation of the arm as well. Showing airflow pattern at different sections in HDD will be helpful for further study on airflow control induced vibration on the HGA.

\section{ACKNOWLEDGEMENT}

This study is supported by Cooperate Project between National Electronics and Computer Technology Center (NECTEC) and Seagate Technology (Thailand) via Industry/University Cooperative Research Center (I/UCRC) in HDD Component, Khon Kaen University.

\section{REFERENCES}

[1] Hirono Y., Arisaka T., Nishijima N., Shimizu T., Nakamura S. and Masuda H., Flow-Induced Vibration Reduction in HDD by Using a Spoiler, IEEE Trans. Magn., vol. 40 No.4, (2004).

[2] Ikegawa M., Hirono Y., Mukai H. and Kaiho M., Decreasing Airflow Velocity in Hard Disk DrivesWith a Spoiler and Bypass, IEEE Transactions on Magnetics, Vol.42, No.10, (2006).

[3] Shimizu H., Shimizu T., Tokuyama M., Masuda H. and Nakamura S., Study of aerodynamic characteristics in hard disk drives by numerical simulation," IEEE Trans. Magn., vol. 37 pp. 831- 863, (2001).

[4] Shiong A. T. C., Suriadi M. A., and Qide Z., Studies on Air Flow Induced Vibration in a simplifiled Hard Disk drive using LES. Asia-Pacific Magnetic Recording Conference, 2006, Page(s):1 - 3.Nov. (2006).

[5] Song H., Damodaran M. and Quock Y. Ng, Simulation of Flow Field and Particle Trajectories in hard disk drives Enclosures, High Performance Computation for Engineered Systems (HPCES), (2004).

[6] Suriadi M.A., Tan C.S., Zhang Q.D., Yip T.H. and Sundaravadivelu K., Numerical investigation of airflow inside a 1-in hard disk drives, Journal of Magnetism and Magnetic Materials 303 (2006).

[7] Takada S., Kusukawa T., Tagawa N., Mori A., Mizoh Y., Nakakita M., Study on flow-induced vibration of head-disk assembly mechanisms in actual hard disk drive," Springer-Verlag., Vol. 13 No. 8-10, (2007). 
FIGURES AND TABLES

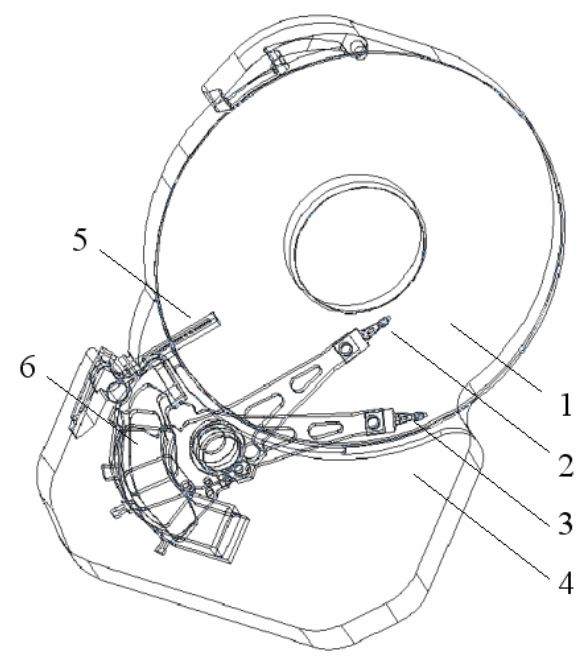

1- Disk; 2- Head at ID position

3- Head at OD position; 4-Base

5- Air Latch; 6- Magnet

Fig.1. Simulation Model of Hard Disk Drive

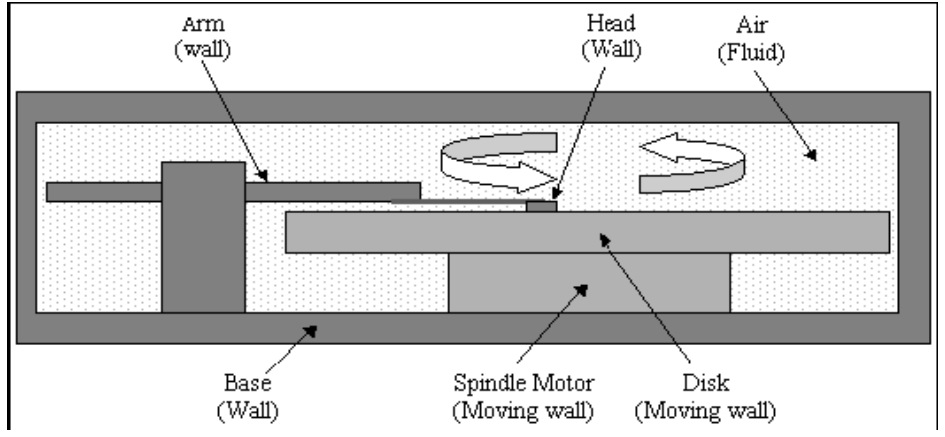

Fig.2. Boundary Conditions

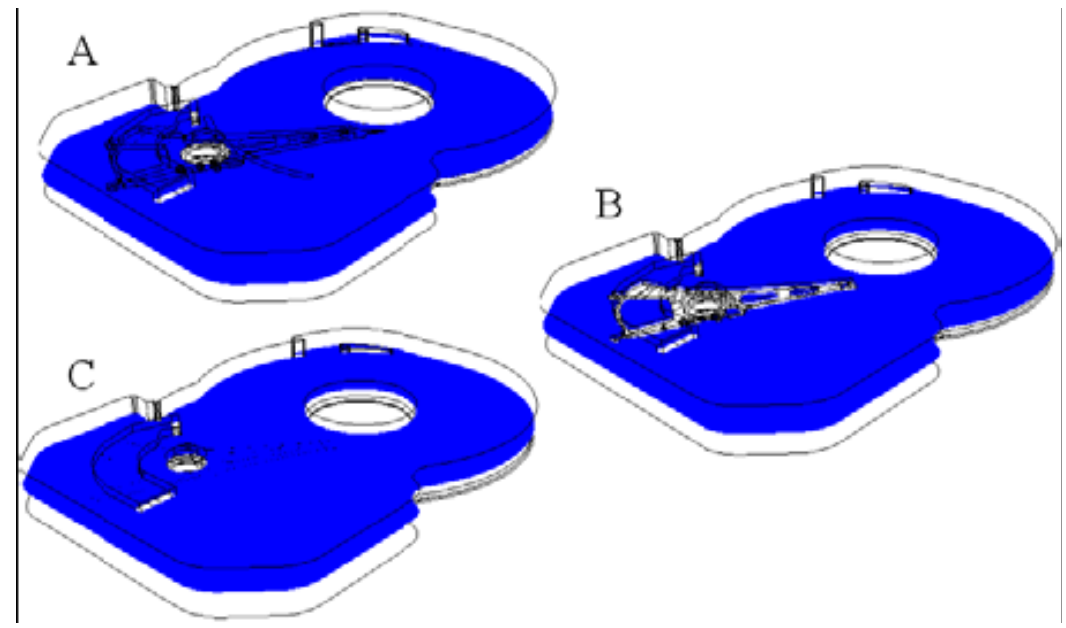

Fig.3. Three Different Horizontal Cut Planes: A-below, B-middle and C-above Actuator Arm 


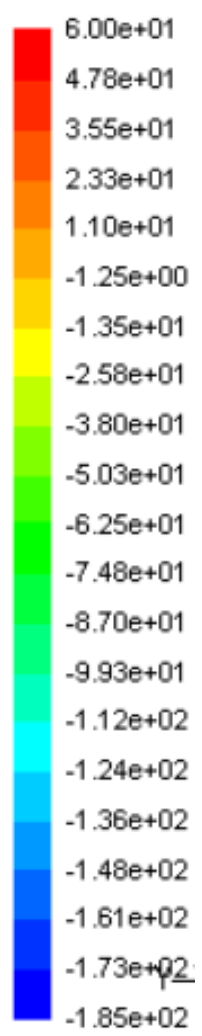

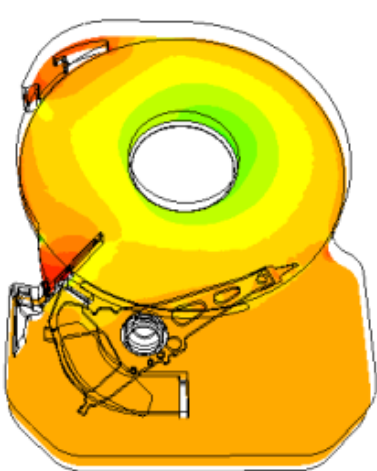

A) OD with air latch

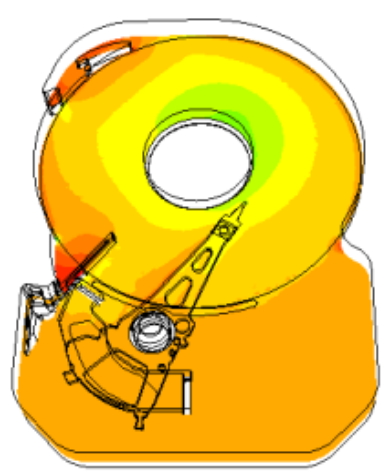

C) ID with air latch

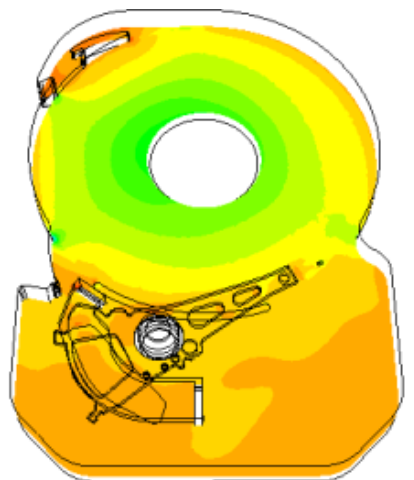

B) OD without air latch

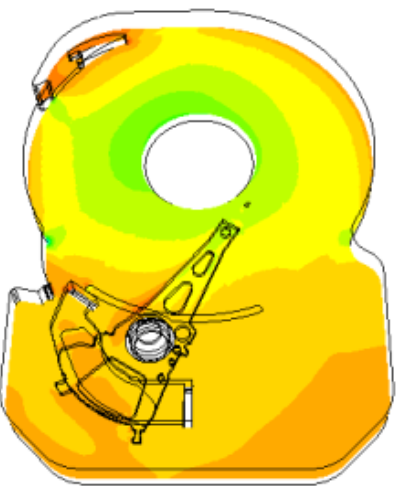

D) ID without air latch

Fig.4. Static Pressure of Cut Plane below Actuator Arm

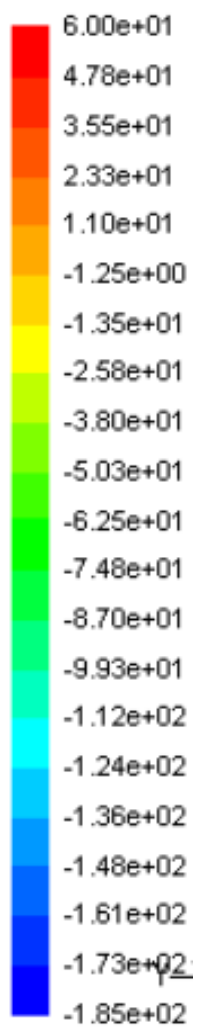

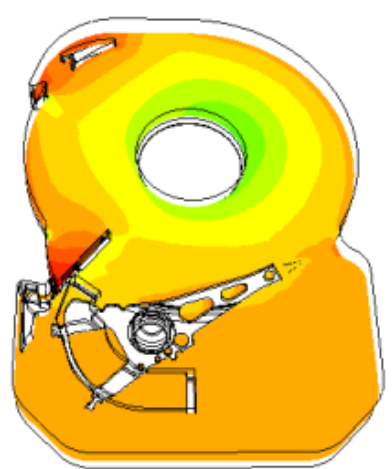

A) OD with air latch

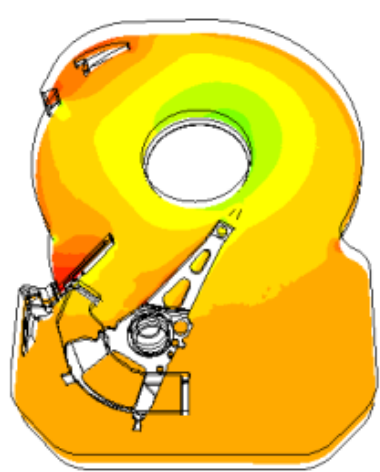

C) ID with air latch

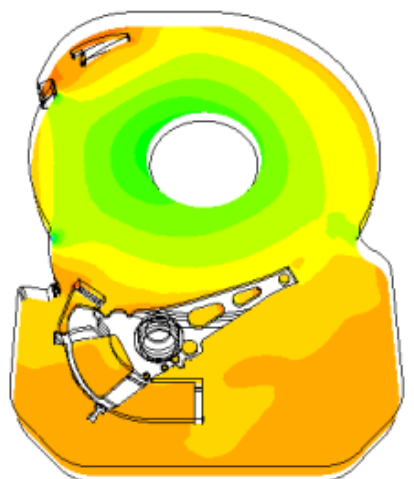

B) OD without air latch

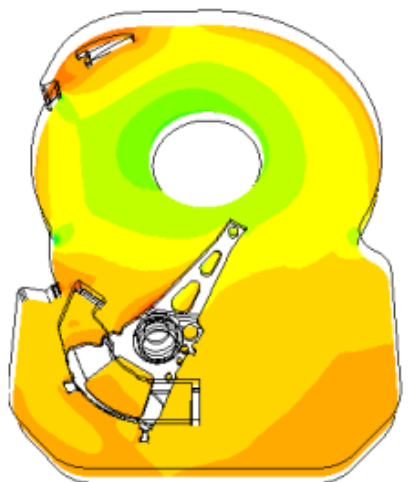

D) ID without air latch

Fig.5. Static pressure of Cut Plane middle Actuator Arm 


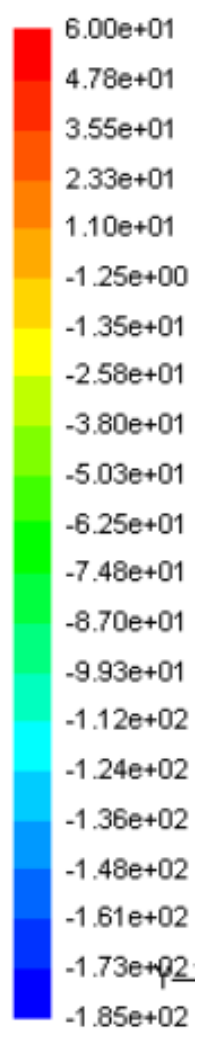

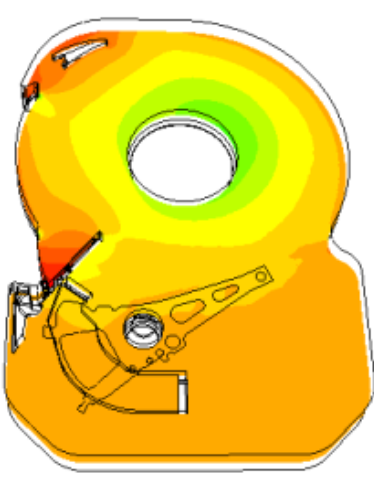

A) OD with air latch

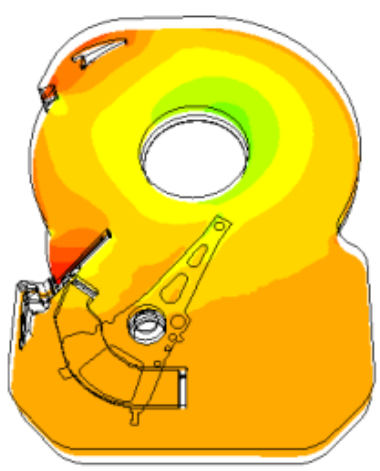

C) ID with air latch

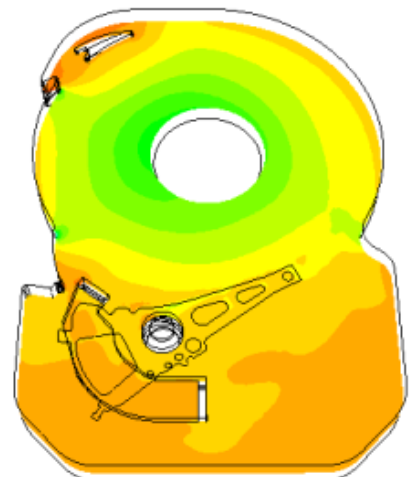

B) OD without air latch

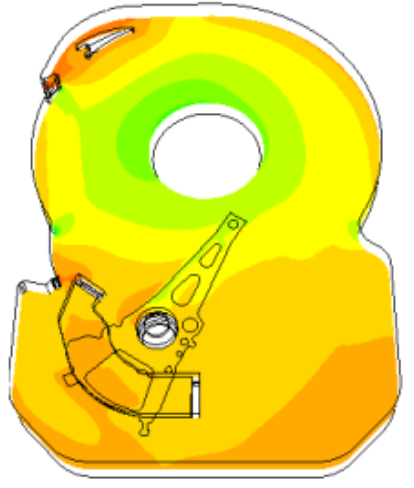

D) ID without air latch

Fig.6. Static Pressure of Cut Plane above Actuator Arm

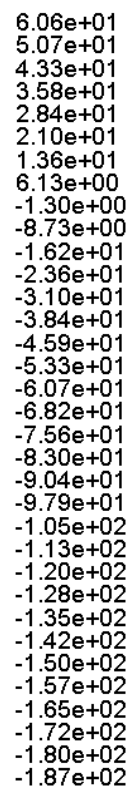

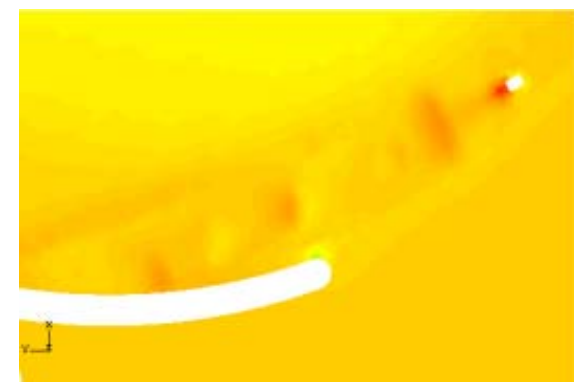

A) OD with air latch

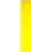

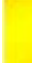

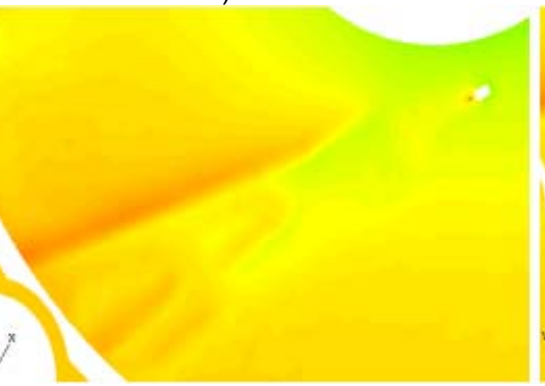

C) ID with air latch

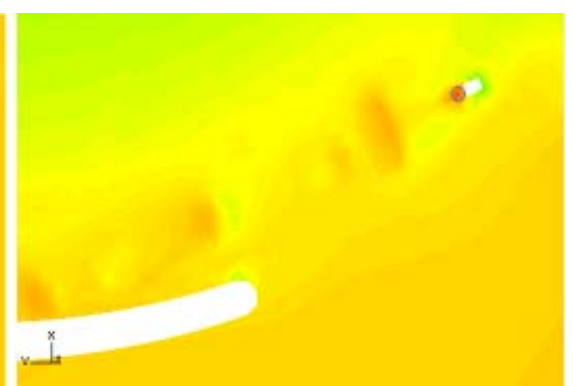

B) OD without air latch

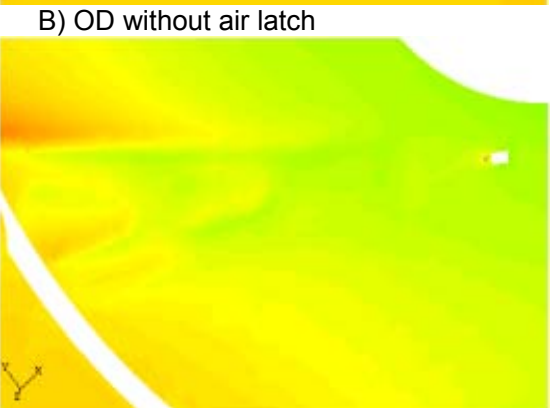

D) ID without air latch

Fig.7. Static Pressure at Recording Head 

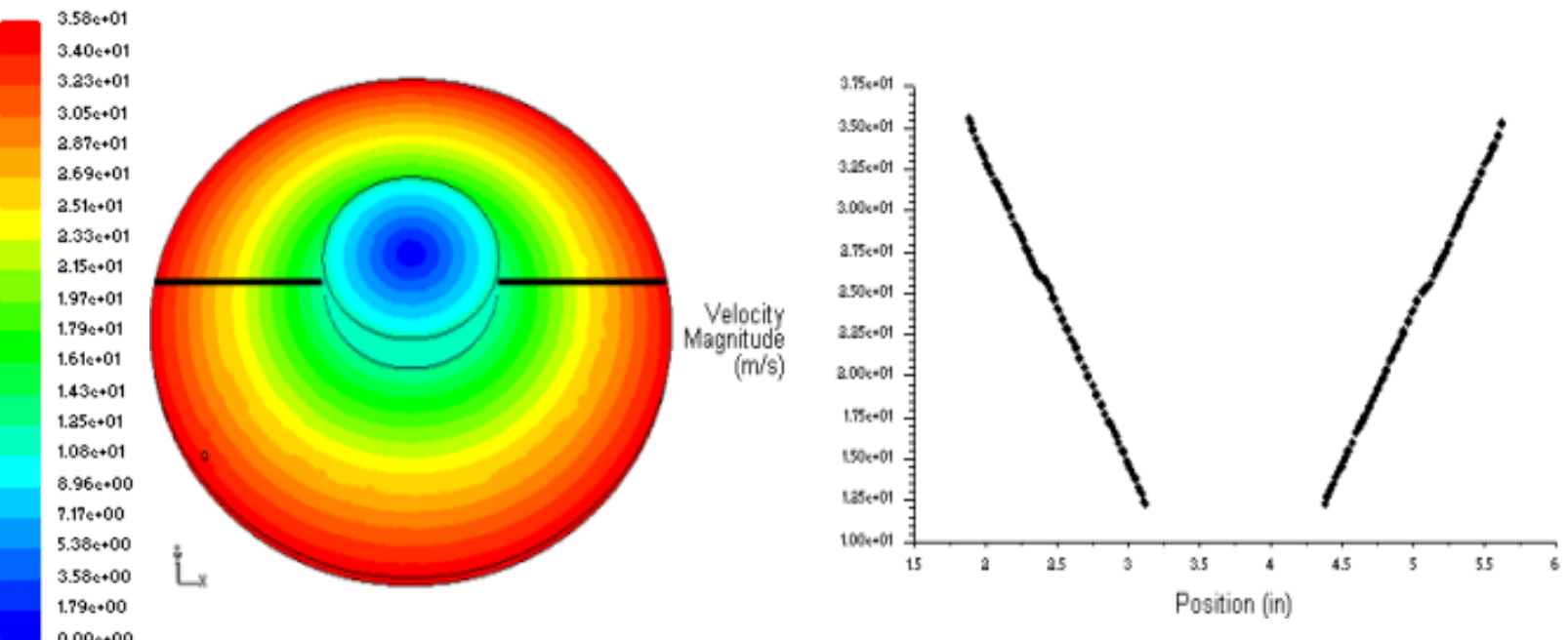

Fig.8. Air Velocity at the Disk Surface.
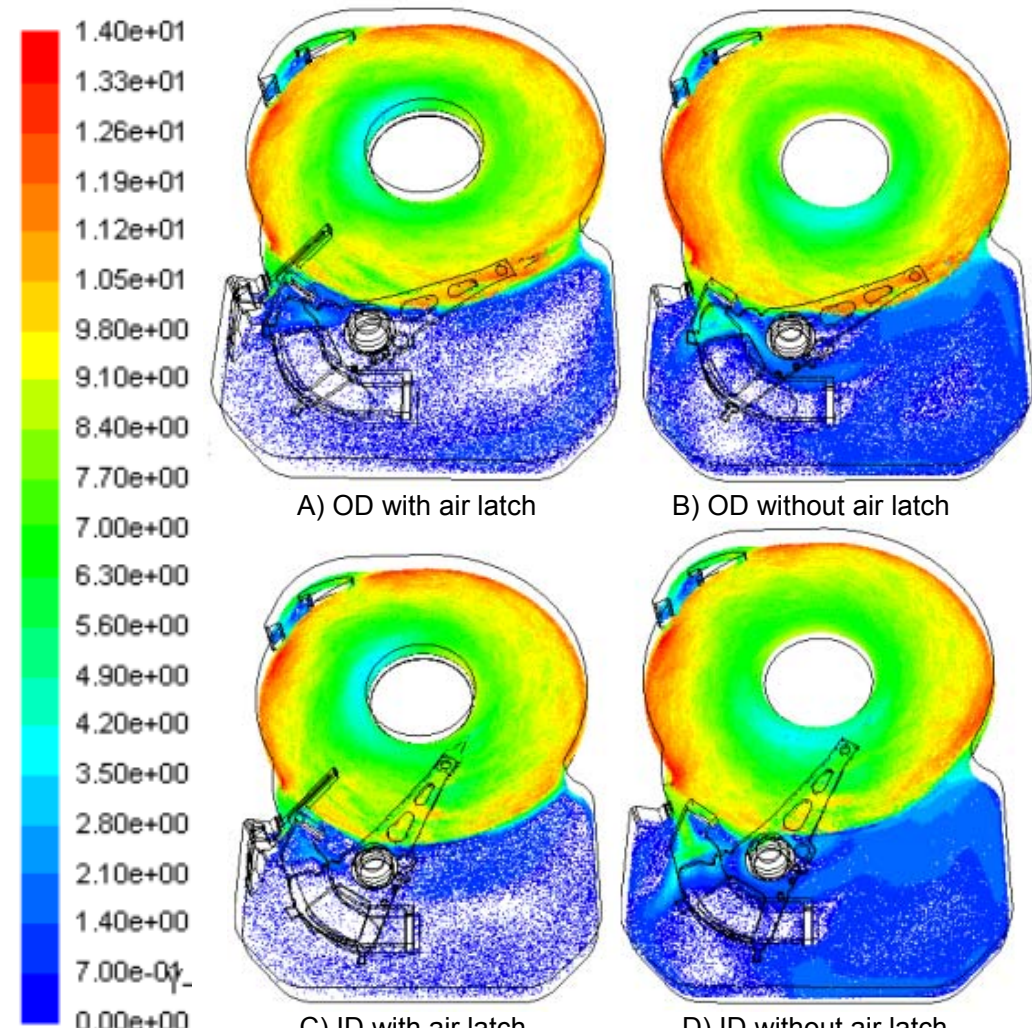

A) OD with air latch

B) OD without air latch
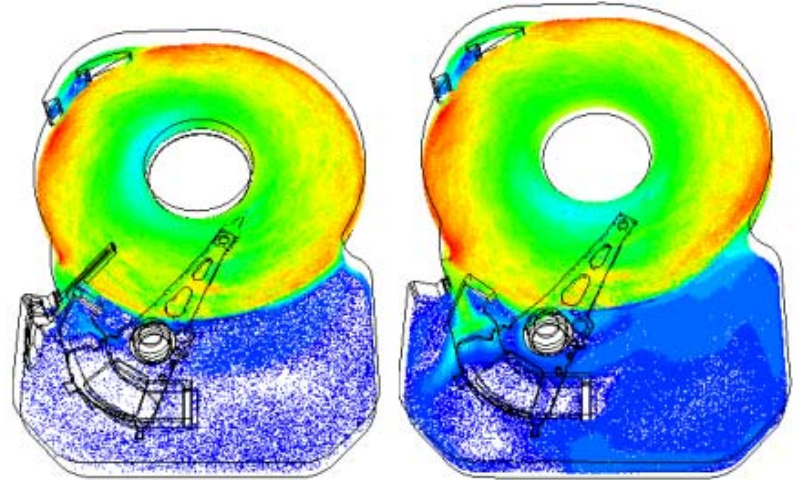

C) ID with air latch

D) ID without air latch

Fig.9. Velocity Magnitude of Cut Plane below Actuator Arm 

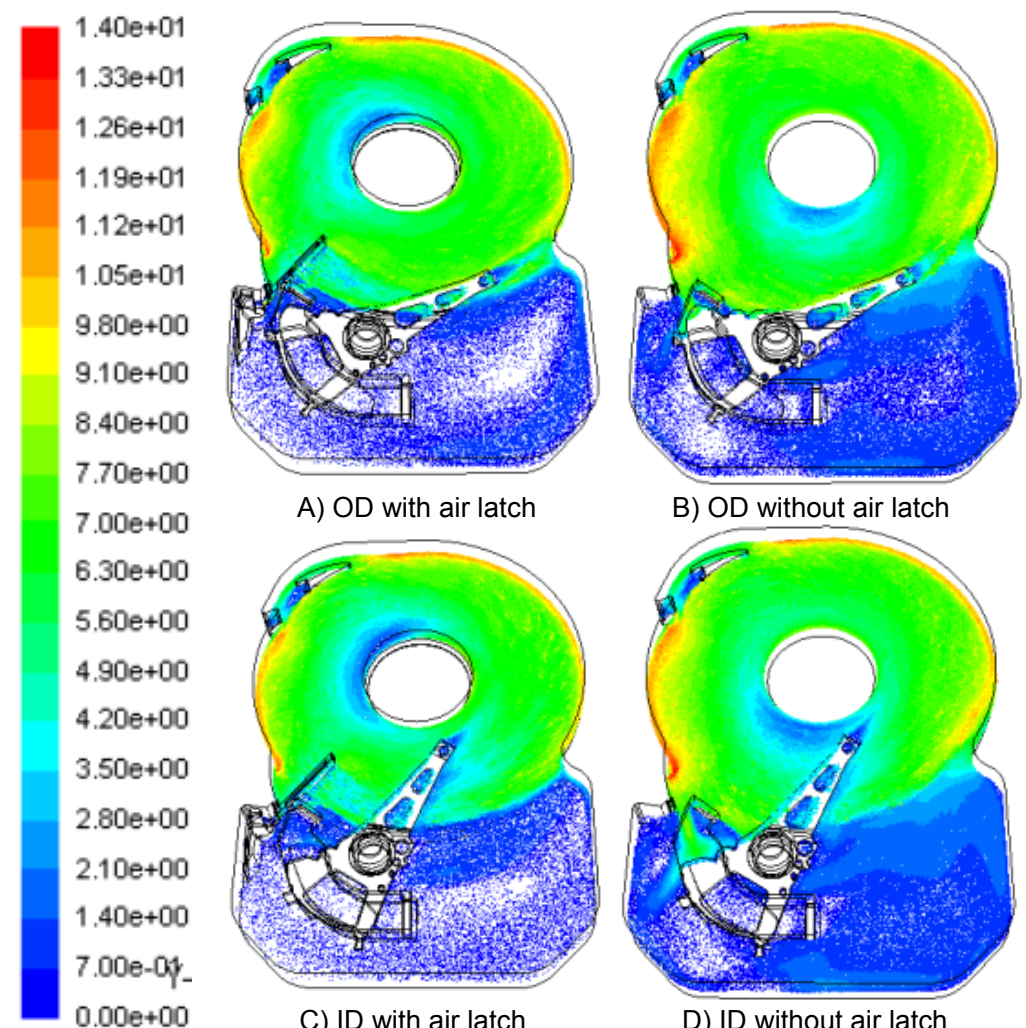

A) OD with air latch

B) OD without air latch
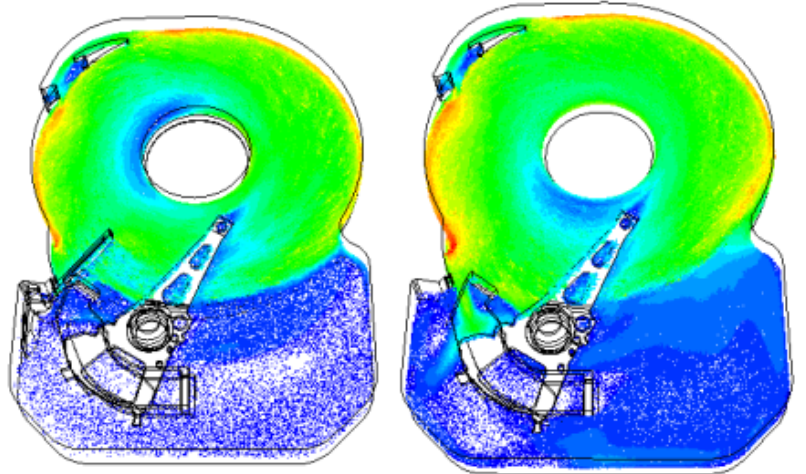

C) ID with air latch

D) ID without air latch

Fig.10. Velocity Magnitude of Cut Plane Middle Actuator Arm.

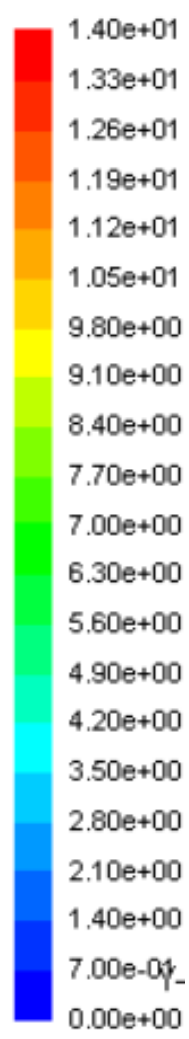

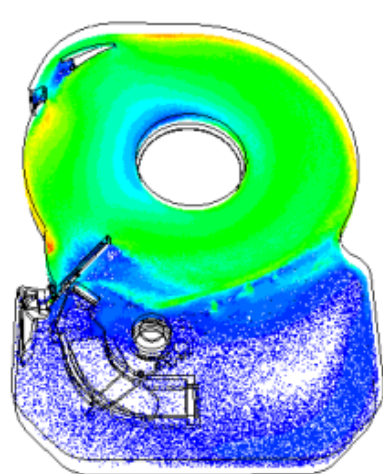

A) OD with air latch

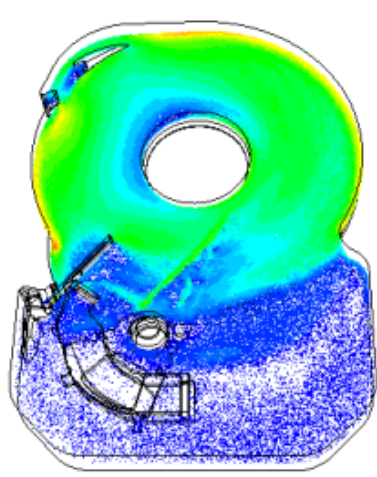

C) ID with air latch

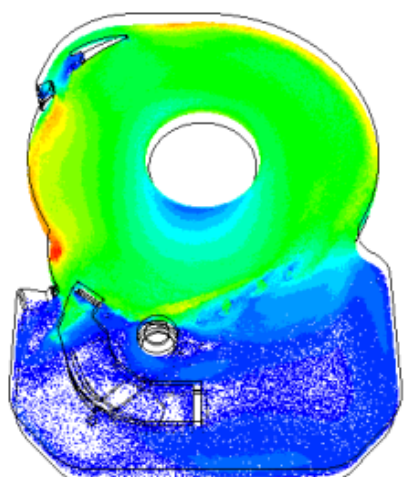

B) OD without air latch

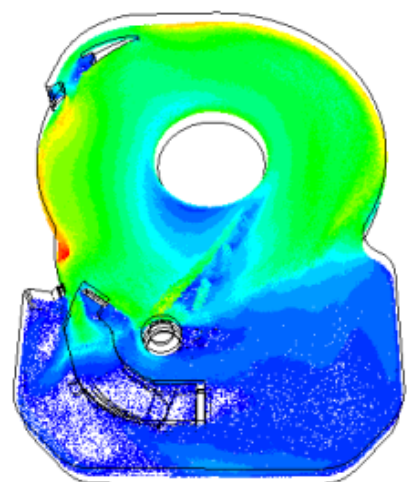

D) ID without air latch

Fig.11. Velocity Magnitude of Cut Plane above Actuator Arm 


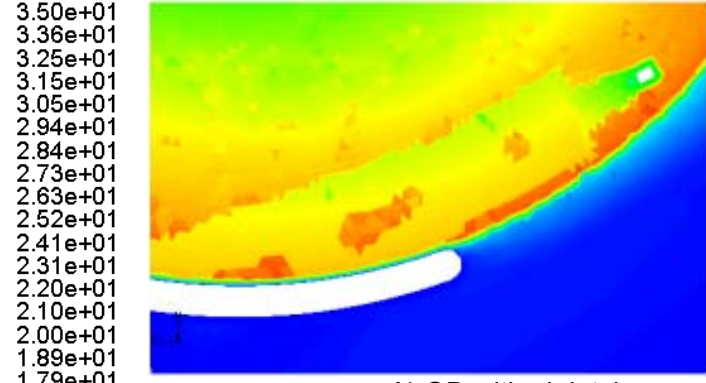

A) OD with air latch

$68 \mathrm{e}+01$

$1.58 \mathrm{e}+01$

$1.47 \mathrm{e}+01$

$1.36 \mathrm{e}+01$

$1.26 \mathrm{e}+01$

$1.16 e+01$
$1.05 e+01$

$9.45 \mathrm{e}+00$

$8.40 \mathrm{e}+00$

$7.35 \mathrm{e}+00$

$7.35 \mathrm{e}+00$
$6.30 \mathrm{e}+00$

$5.25 \mathrm{e}+00$

4.

$3.15 \mathrm{e}+00$

$1.105+00$

$0.00 \mathrm{e}+00$

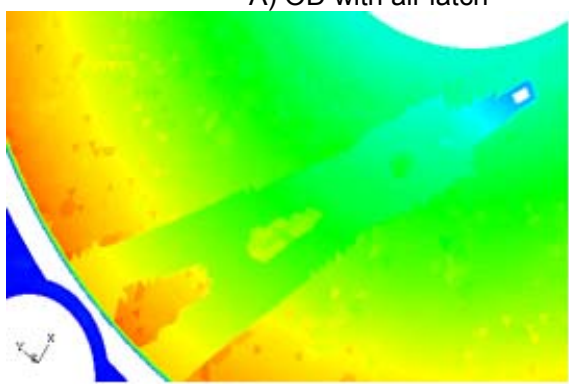

C) ID with air latch

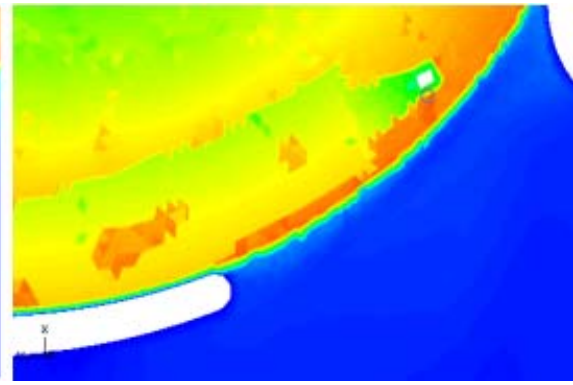

B) OD without air latch

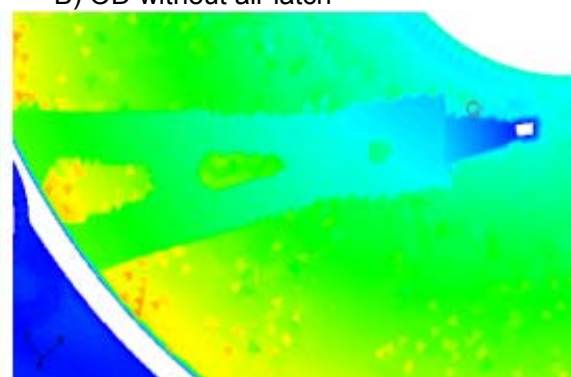

D) ID without air latch

Fig.12. Magnitude of Velocity at Actuator Head.
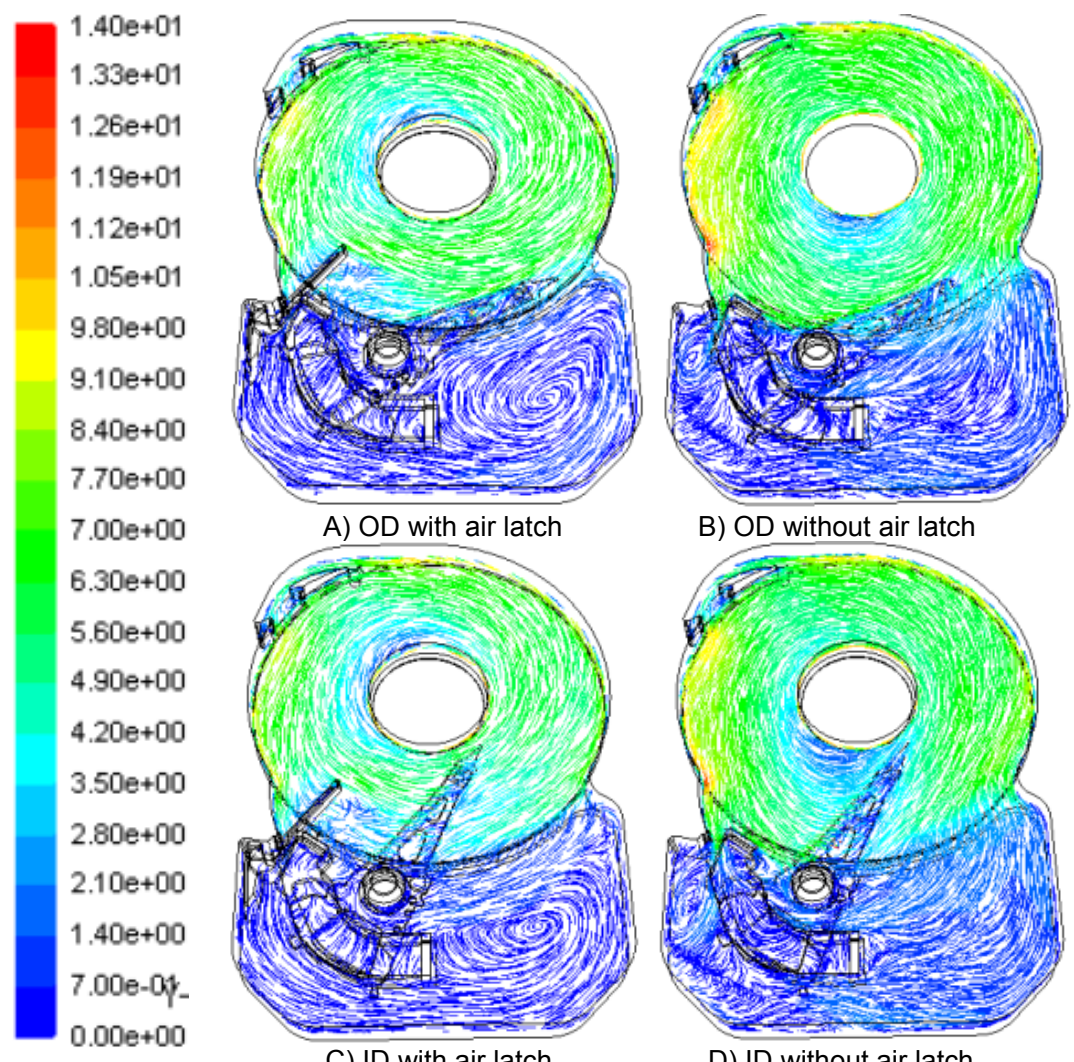

A) OD with air latch

B) OD without air latch
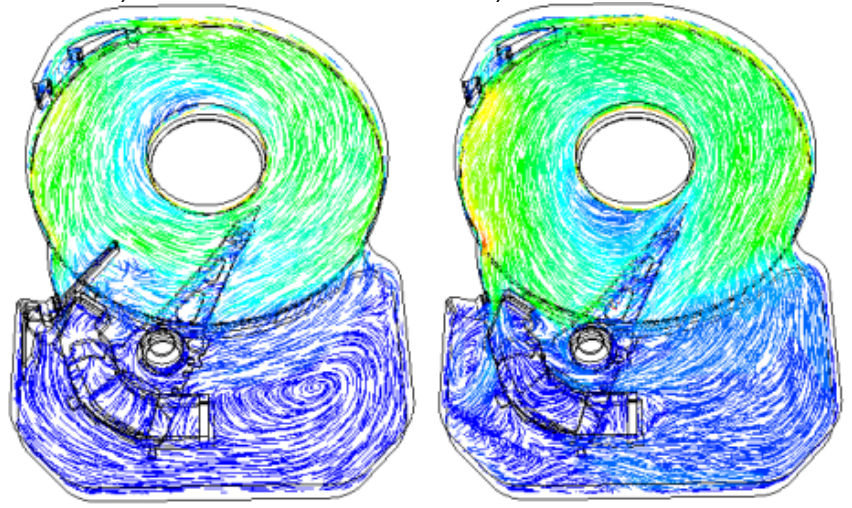

C) ID with air latch

D) ID without air latch

Fig.13. Flow Pattern between OD and ID Actuator Head Position on Horizontal Plane above Actuator Arm 


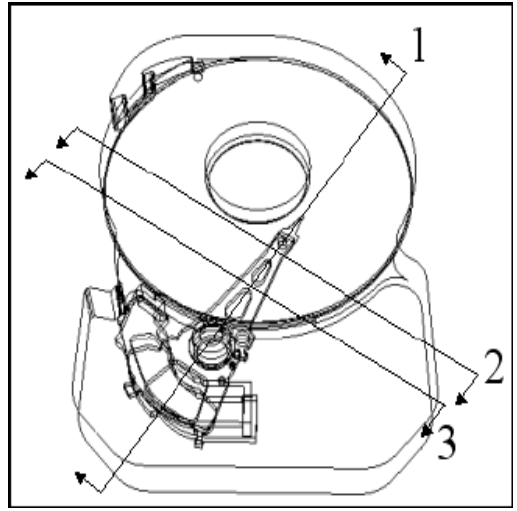

1. Cutting along actuator arm.

2. Cutting at the tip of actuator arm.

3. Cutting at the middle of actuator arm.

Fig.14. Vertical Cut Plane.
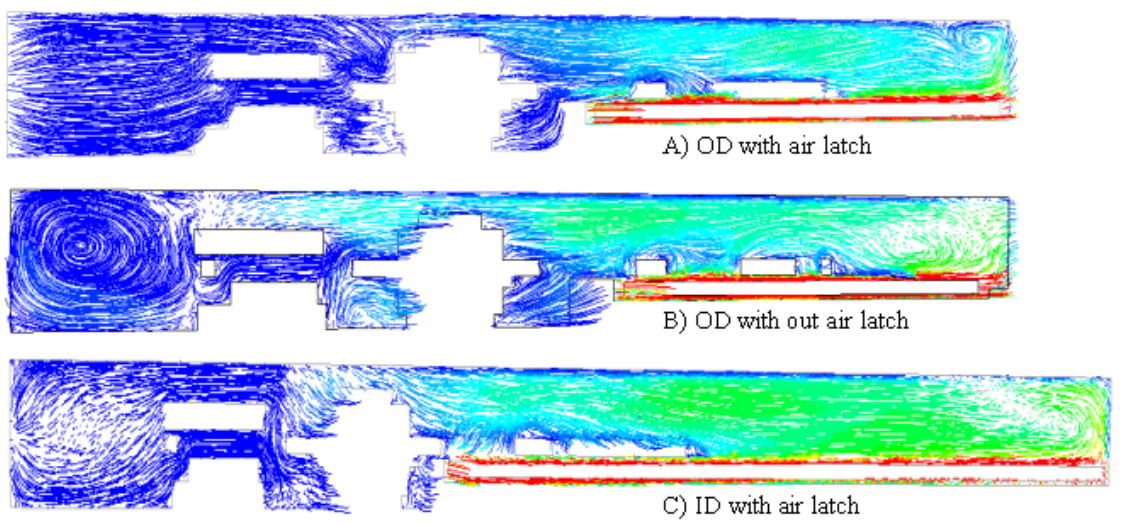

C) ID with air latch

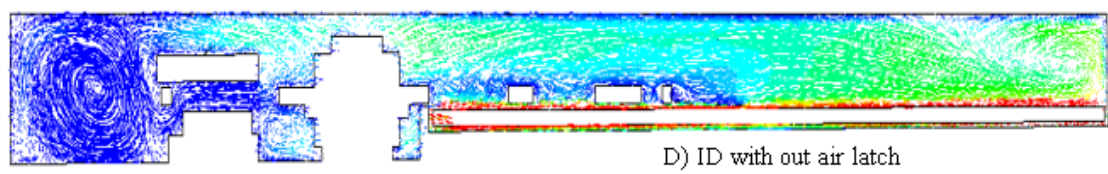

Fig.15. Flow Pattern on Cut Plane along Actuator Arm
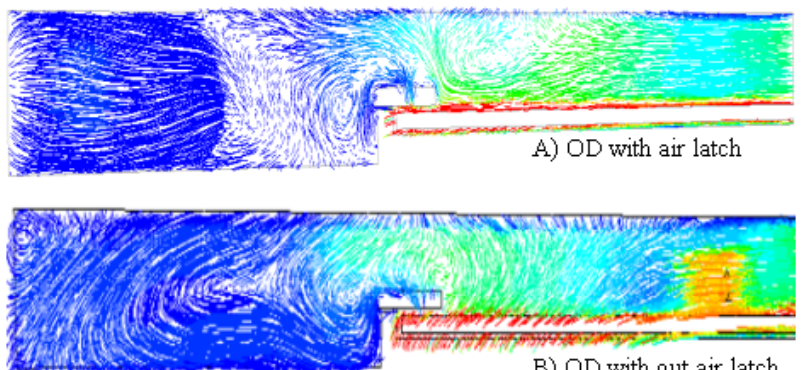

B) OD with out air latch

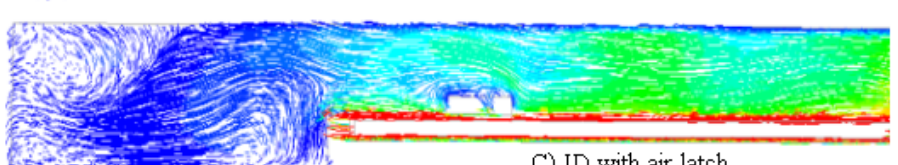

C) ID with air latch

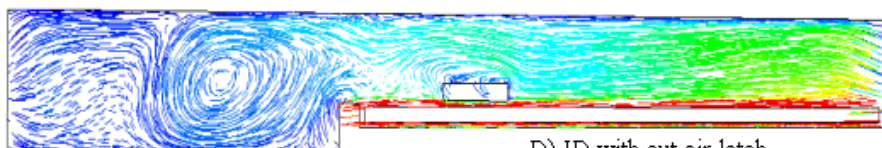

D) ID with out air latch

Fig.16. Flow Pattern on Cut Plane at Middle Actuator Arm 


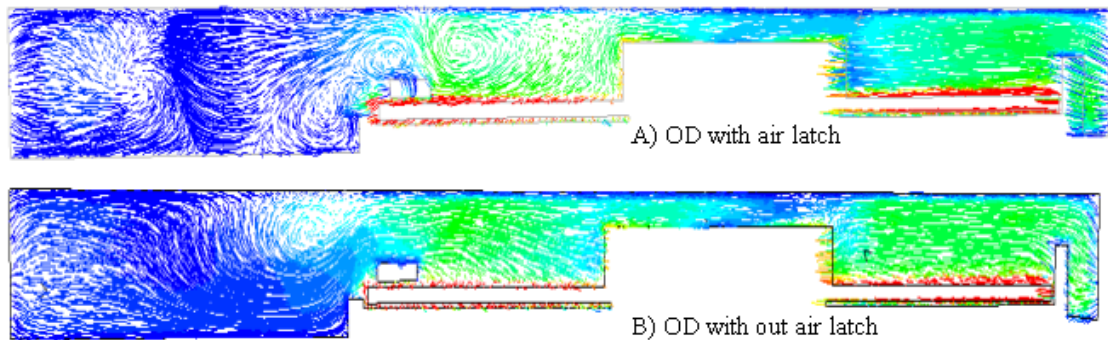

B) OD with out air latch

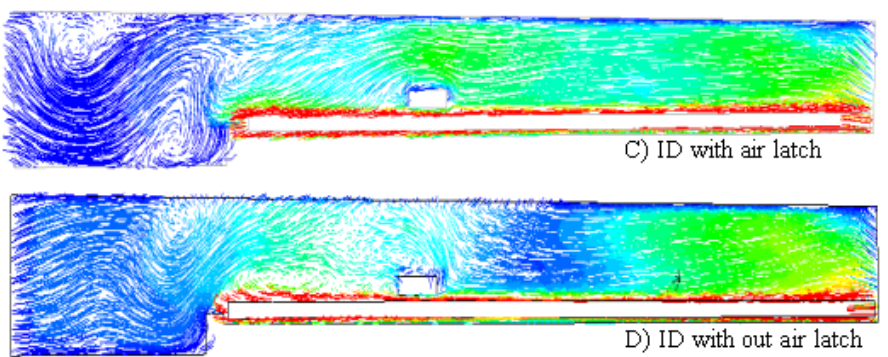

Fig.17 Flow Pattern on Cut Plane at Tip of Actuator Arm

Table 1. Comparison of Maximum Static Pressure at Recording Head.

\begin{tabular}{|c|c|c|}
\hline Head Position & $\begin{array}{c}\text { Maximum static pressure at } \\
\text { recording head (With air latch) }\end{array}$ & $\begin{array}{c}\text { Maximum static pressure at } \\
\text { recording head (Without air latch) }\end{array}$ \\
\hline ID & $-1.3 \mathrm{pa}$ & $-16.2 \mathrm{pa}$ \\
\hline OD & $50.7 \mathrm{pa}$ & $43.2 \mathrm{pa}$ \\
\hline
\end{tabular}

Table 2. Comparison of Maximum Velocity at Recording Head

\begin{tabular}{|c|c|c|}
\hline Head Position & $\begin{array}{c}\text { Maximum velocity at recording } \\
\text { head (With air latch) }\end{array}$ & $\begin{array}{c}\text { Maximum velocity at recording } \\
\text { head (Without air latch) }\end{array}$ \\
\hline ID & $11.5 \mathrm{~m} / \mathrm{s}$ & $10.8 \mathrm{~m} / \mathrm{s}$ \\
\hline OD & $31.0 \mathrm{~m} / \mathrm{s}$ & $31.2 \mathrm{~m} / \mathrm{s}$ \\
\hline
\end{tabular}

